\title{
A Condition for Complete Flattening of Asperities in a Rough Contact
}

\author{
Mikhail V. Murashov ${ }^{1}$ \\ ${ }^{1}$ Bauman Moscow State Technical University, 105005 Moscow, Russia
}

\begin{abstract}
Considering rough surface profiles in a contact model is of decisive importance. In the up-to-date rough contact models there remained underexplored the opportunity of complete flattening of smaller asperities and therefore the need of using the multilevel roughness models, including fractal ones. If higher level asperities are not flattened completely when pressed, then they will be able to impact on the contact process. This paper considers model problems of elastic-plastic contact with hardening for a body with protrusions and two pyramids as the objects similar to asperities. Modeling results show that asperities are completely flattened only on condition of confined compression. For real contacting rough surfaces under low pressures, complete flattening of asperities will not occur. It is shown that roughness elements on the surface of the asperities do not disappear even at severe deformation of the latter. The reason is a combination of the asperity form and hardening of material, while the consequence is a reduction of the real contact area.
\end{abstract}

\section{Introduction}

The problems relating to static and dynamic contact of solid bodies play an important role in such fields as tribology, heat transfer, electrodynamics, welding, and also in automobile, space, nuclear and precision instruments industries. Considerable issues emerge at describing the contacts in such intensively developing areas as micro- and nanotechnologies, especially at design of micro-electro-mechanical systems.

At the beginning of the 20th century, a great influence of rough contacts on the thermal state of some assembled structures was discovered. Real contact area of rough surfaces makes only a small share (usually less than $1 \%$ ) from the nominal area outlined by geometrical dimensions of the adjoining bodies. This circumstance determines decrease in thermal contact conductance, the assessment of which was always a problem area in researches on contact heat transfer [1]. So far, it is not developed a reliable technique for quantitative prediction of the parameters of contact heat transfer. Capabilities of experiment in this case are limited and insufficient [2]. The model yielding the most reliable results on contact heat transfer is mainly necessary at the design stage of future device.

There are systems requiring the temperature prediction with high accuracy, for example, navigation systems for launch vehicles [3] and similar devices of precision instruments industry. To meet these requirements, the thermal contact model has to consider the roughness structure of contacting surfaces and contact heat transfer features in the micron-sized contact zone. It becomes necessary to model deformation of separate roughness elements (asperities)

Thus, there is a question whether asperities flatten completely at the contact of rough surfaces. Under what conditions is it possible? There are smaller asperities based on the surface of bigger ones and called asperities of higher level. These asperities are included in widely adopted multilevel [4] and fractal [5-12] 3D models of roughness. The additional question is specific for such models - whether preservation of rough profile on an asperity surface is possible while deforming by a rigid plane i.e. whether asperities of higher level will be flattened completely first at deformation? If yes, then at certain values of contact pressure the asperities of a higher level could be neglected, thus considerably simplifying the contact model. Otherwise, if no preliminary flattening of higher level asperities occurs, then the roughness model in the contact model is rather to be multilevel. Then, the adequate picture of rough surfaces deformation including that for the real contact area determination could be obtained through numerical modeling, for example, with the use of finite element method.

To date a number of models of thermal contact conductance through multi-spot contact are developed, for example, see [7,13-16], from which it is possible to distinguish finite element models $[7,15,16]$. It is to finiteelement models that they are increasingly turning to validate analytical stochastic models, for example, [1720]. Finite element modeling of rough surfaces contact is considered to be superior for elastic-plastic material behavior [21] and, moreover, is expected to deliver reference results for other contact mechanics approaches 
[17]. The essential advantage over other methods is also that it allows to consider influence of the form of asperities and change of material properties in the deformation process.

These advantages seem important, because, for example, Greenwood [22] has already shown (for electric contact) that contact resistance is defined not only by number and size of individual contact spots, but also by distance between them and regularity of their arrangement. Besides, it is easy to confirm experimentally (see, for example [23]) that under the pressure of a flat polished punch applied to a rough surface, the notches, grooves and all the more cracks remain on the surface even at considerable plastic deformation of the whole body. The rough profile of a print after Brinell hardness indentation test is presented in [22]. In addition, Kochergin [24] shows residual rough profile of aluminum surface after pressing at $200 \mathrm{MPa}$ with Johansson gauge block. The indirect evidence of the profile flattening inability is the indication, reproduced in a range of works (for example, in [25]), of decrease in the real contact area with increase in scatter of the asperities heights.

To answer the raised questions, this paper considers solution of some model problems in finite element software ANSYS intended to elucidate the role of surface form in contact deformation of roughness. The problems are solved in an elastic-plastic formulation with strain hardening, inclusion of which in contact models is the problem of great importance [26]. Section 2 considers the model problem of elastic-plastic flattening of a single protrusion on a surface for clarification of the influence of the asperities presence on surfaces interaction and, therefore, on the size of the real contact area at finite element modeling. The condition is defined under which complete flattening is possible. Section 3 considers the model problems of upsetting a regular square and a doubled truncated pyramids as models of asperities. These models have been chosen taking in mind deterministic approach to rough surface description. The models have to be $3 \mathrm{D}$ and cannot be considered as $2 \mathrm{D}$ as they have variable thickness. The section shows features of deformation defined by a pyramid form and presence of hardening. The doubled pyramid with a valley at top is considered to be a model of an asperity containing asperities of the second level. The obtained result shows that the valley is not smoothed even at a severe deformation of the whole pyramid. Discussion of obtained results is given in section 4 .

Note that the problem of deformation of single asperity is considered here not for the first time. In an elastic formulation, in fact, it was solved by Hertz in 1882 in the problem of the contact of two parabolic bodies [27]. But a purely elastic contact of the asperities is impossible, since all calculations and a series of experiments give stresses at the asperities much exceeding the yield strength of the material [28,29]. Therefore, a lot of work is devoted to single asperity models. More often, three approaches were used: the flattening of the deformed sphere between two absolutely hard surfaces [30-32], the flattening of the sinusoidal asperity by a rigid flat $[21,28,29]$, and the indentation of a rigid hemisphere into the deformable space. Ghaednia et al. [33], using the finite element method, solved a thermo-electro mechanical axisymmetric problem for elastic perfectly-plastic deformation of a hemisphere and a substrate. A FEM solution and a comparison with experiment are given by Wadwalkar et al. [34] for the problem of deformation of a sphere between two rigid flat surfaces in the case of large pressures (up to $2.6 \mathrm{GPa}$ ). Modeling of an elastic perfectly-plastic creep deformation of a hemispherical asperity is carried out by Goedecke et al. [35].

But the aim of these and similar works was to obtain simple relations for inclusion in multi-spot statistical contact models [36,37], including those that take into account the interaction of asperities [38-42] and hardening [28]. However, in these works there is no answer to the question of whether a complete flattening of the asperities is possible. This study aims at clarifying issues that arise not in statistical contact models, but in finite-element spatial modeling of contact, when the individual geometry of each asperity is specified. For this case, the adopted models of asperities in the form of pyramids seems to be more suitable for modeling real roughness than hemispheres. Moreover, a deformation of a single asperity with a defect in shape (a valley at top), to the extent of the authors knowledge, has not previously been considered.

While solving the problems we assume lack of initial cold work hardening, residual stresses and influence of indentation size effect [43] considerably strengthening the surface when penetrating to several microns [44]. To obtain general patterns of asperities' behavior at flattening, carrying out calculations within assumptions of continuum mechanics, we accept the sizes of the bodies, the protrusion and the valley to be macro-scale. Static analysis is used, that is the deformation happens in the isothermal mode and does not depend on loading speed. The flow theory of plasticity and additive approach to forming of strain increments are applied.

\section{Modeling the flattening of surface protrusions}

To clarify the opportunity of complete flattening of asperities we consider the following model problem. Let us compare behavior of two bodies at compression. The first body has a flat upper surface. The second has protrusions on the upper surface (see Figure 1), imitating asperities.



Figure 1. The surface of the second body with protrusions. The broken line shows the area taken for symmetry reasons in finite element model.

Due to the symmetry the first and the second body can be represented by models of parallelepipeds 
$0.05 \times 0.05 \times 0.5 \mathrm{~m}$ in size (see Figure 2). The upper surface of the second body has a protrusion in the form of a rectangular parallelepiped of $0.03 \times 0.03 \times 0.01 \mathrm{~m}$ in size. The Cartesian coordinate system axes are directed as shown in Figure 2.



(a)

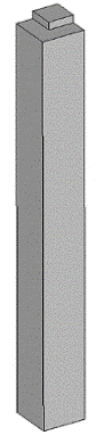

(b)

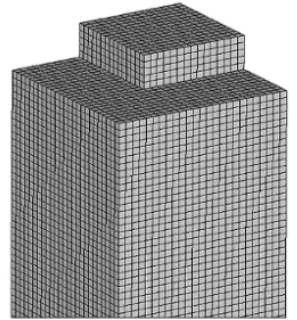

(c)
Figure 2. Geometrical models of the first (a) and the second (b) bodies and a finite element model of the upper part of the second body (c).

Material of the bodies is copper type UNS C12500 ASTM B224-16 (the modulus of elasticity $120 \mathrm{GPa}$, Poisson's ratio 0.38). It is deformed elastically and plastically with isotropic hardening. The stress-strain curve for the material [45] was recalculated for logarithmic strains and approximated with multilinear model (see Figure 3). As the last point in the experimental loading curve is a stress of about $375 \mathrm{MPa}$ at logarithmic strain 0.7 , then to make the possibility to calculate at a bigger strains the multilinear curve was continued by means of linear extrapolation to the point with logarithmic strain equal 2 (flow stress of $570 \mathrm{MPa}$ ). After this point the material flows as rigid-perfectly plastic.

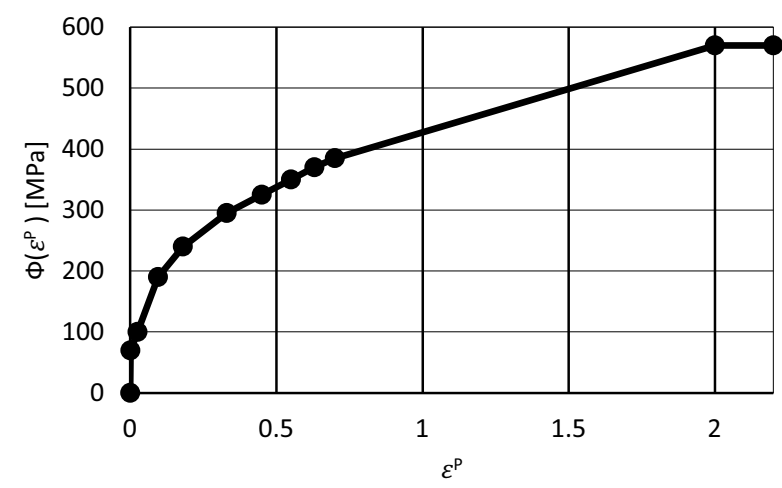

Figure 3. The model of the stress-strain curve for copper UNS C12500 at compression.

The punch in the form of a parallelepiped of $0.3 \times 0.3 \times 0.1 \mathrm{~m}$ exerts pressure $P$ on the upper surfaces of bodies. To consider the deformation of the punch insignificant its material is assumed to be hypothetical, elastic, with the modulus of elasticity of $E=2 \cdot 10^{18} \mathrm{~Pa}$ and Poisson's ratio $v=0.3$. The lower surface is fixed to avoid displacement. Lateral surfaces are fixed to avoid displacement in the directions orthogonal to them. The friction is neglected in the solution.

Let us consider a 3D quasi-static mathematical model of contact between the plate of $V_{1}$ volume with $S_{1}$ surface and one of the two bodies of $V_{2}$ volume with $S_{2}$ surface. We designate the upper surface of the plate as $S_{1}^{1}$ and the rest of the surface as $S_{1}^{2}$, so that $S_{1}=S_{1}^{1} \cup S_{1}^{2}$. For the body chosen we allocate in the surface $S_{2}$ an upper surface (together with protrusion, if any) $S_{2}^{1}$, the lower surface $S_{2}^{3}$, and the rest of the surface $S_{2}^{2}$, so that $S_{2}=$ $S_{2}^{1} \cup S_{2}^{2} \cup S_{2}^{3}$.

The surface $S_{1}^{1}$ is affected by the external pressure $P$. The mathematical model comprises equilibrium equations (1), the generalized Hooke's law (2), the flow rule (3), strain-displacement relations (4), von Mises yield condition (5), ratio for calculation of contact pressure of augmented Lagrangian method (6), and boundary conditions (7)-(9)

$$
\begin{gathered}
\sigma_{j i, j}=0 ; \\
\varepsilon_{i j}=\frac{1+v}{E} \sigma_{i j}-\frac{v}{E} \delta_{i j} \sigma_{k k} ; \\
d \varepsilon_{i j}^{P}=s_{i j} d \lambda ; \\
\varepsilon_{i j}=\frac{1}{2}\left(u_{i, j}+u_{j, i}\right) \\
\left(\sigma_{1}-\sigma_{2}\right)^{2}+\left(\sigma_{2}-\sigma_{3}\right)^{2}+\left(\sigma_{3}-\sigma_{1}\right)^{2}=2 \Phi\left(\varepsilon^{P}\right)^{2} ; \\
p\left(x_{i}\right)=K \delta+\lambda_{c}, x_{i} \in S_{2}^{1} ; \\
\sigma_{33}=P, x_{3} \in S_{1}^{1}, \\
u_{1}\left(x_{2}\right)=0, u_{2}\left(x_{1}\right)=0, x_{1}, x_{2} \in S_{2}^{2}, \\
u_{3}\left(x_{3}\right)=0, x_{3} \in S_{2}^{3} .
\end{gathered}
$$

where $\sigma_{i j}$ and $\varepsilon_{i j}$ are the Cartesian components of tensors of stress and strain, $u_{i}$ - components of displacements vector, $E-$ the modulus of elasticity, $v$ - Poisson's ratio, $\delta_{i j}-$ Kronecker delta, $s_{i j}-$ components of stress deviator tensor, $\sigma_{1}, \sigma_{2}, \sigma_{3}-$ the principal stresses, $\lambda, \lambda_{c}$ - Lagrange multipliers, $\Phi\left(\varepsilon^{P}\right)$ - function of the material's stressstrain curve, $p$ - the contact pressure, $P$ - external pressure applied to the punch, $K$ - contact stiffness, $\delta-$ contact gap size.

After preliminary calculations it was obtained that the second body's protrusions are completely flattened at external pressure of $15 \mathrm{MPa}$, and this requires defining two pairs of contacting surfaces in the finite element model:

1) the upper planes of the body and protrusion TARGE170 elements; the lower surface of the punch CONTA174 elements;

2) the lateral surfaces of the protrusion - TARGE170 elements; the upper plane of the body - CONTA174 elements.

The model is meshed with $3 \mathrm{D}, 20$-node hexahedral finite elements of SOLID186 type. SOLID187 and SOLID285 elements can be also used for solution. The mesh of the second body consists of 44875 SOLID186 elements.

Rigid fixing of the lower plane of the bodies is admissible in such model elastic-plastic problem, but it disallows comparing results at high pressures with those of real constructions (especially on $x_{3}$-axis). When modeling near-surface volumes of real constructions with columnar models similar to those provided in $[7,46]$, the adequate deformation of the column volume is possible if stress $\sigma_{33}$ on the lower plane do not exceed the initial elastic limit of the material used. 
The results of calculations are reported in the Table 1 and in Figure 4, 10.

Table 1. The results of calculations of the bodies deformation at $P=15 \mathrm{MPa}$.

\begin{tabular}{|c|c|c|c|c|c|c|c|c|}
\hline Model & \multicolumn{2}{|c|}{$\begin{array}{c}\text { Equivalent } \\
\text { stress, MPa }\end{array}$} & \multicolumn{2}{|c|}{ pquivalent } & \multicolumn{2}{|c|}{$\begin{array}{c}\text { Equivalent } \\
\text { elastic strain strain }\end{array}$} & $\begin{array}{c}\text { Punch } \\
\text { displacement } \\
\text { on } \boldsymbol{x}_{\mathbf{3}} \text {-axis, } \\
\text { mm }\end{array}$ & $\begin{array}{c}\text { Maximum } \\
\text { contact } \\
\text { pressure, } \\
\text { GPa }\end{array}$ \\
\cline { 2 - 6 } & $\mathbf{m a x}$ & $\mathbf{m i n}$ & $\mathbf{m a x}$ & $\mathbf{m i n}$ & $\mathbf{m a x}$ & $\min$ & & 0.54 \\
\hline First body & 71.4 & 71.4 & 0.0014 & 0.0014 & 0.0006 & 0.0006 & 1.52 & 0.91 \\
\hline $\begin{array}{c}\text { Second } \\
\text { body }\end{array}$ & 570 & 31 & 2.167 & 0 & 0.0048 & 0.0003 & 7.78 & 2.91 \\
\hline
\end{tabular}

In case of uneven tri-axial compression, the deformation under the set pressure occurs elastically and plastically. The plastic strain is calculated on the basis of the von Mises distortion energy theory without taking into consideration the elastic volume change that is the usual approach to a deformation model of metals $[47,48]$. At the same time the volume of the columnar model decreases because of confined elastic compression. The material is redistributed due to plastic deformation in the zones which are set free because of elastic compression. Then, plastic strain on $x_{3}$-axis (for the second body out of the area near the protrusion) is equal to the sum of elastic strains on axes $x_{1}$ and $x_{2}$. Complete flattening is possible only in the presence of tri-axial elastic compression. It means for columnar models that in the absence of noticeable elastic strain on axes $x_{1}$ and $x_{2}$ complete flattening is impossible. In the calculations made the elastic strains on axes $x_{1}$ and $x_{2}$ on the lower surface $S_{2}^{3}$ of the first and second bodies appeared beginning with $P$ $=5 \mathrm{MPa}$.

It is not possible to forecast the pressure value of complete flattening of asperities without the use of numerical calculations yet. In general case due to uneven profile of the surface complete asperities flattening state is reached only at completely close contact equivalent to perfect one.

Equivalent von Mises stress in the first body is equal to $71.4 \mathrm{MPa}$ and is constant in all volume $V_{2}$. Von Mises stress on the lower surface of the second body $S_{2}^{3}$ is also equal to $71.4 \mathrm{MPa}$. However, because of uneven upper surface, a non-uniform distribution of stresses and strains in the volume $V_{2}$ appears. Areas of both higher and lower stresses and strains exist, and the area with lower strains is the layer of less hardened material lying in some depth from the surface (see Figure 4).

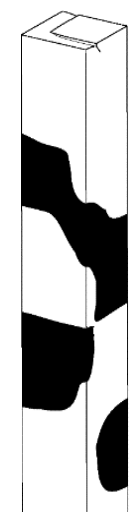

Figure 4. The area of the second body where equivalent plastic strain does not exceed the value for first body (shown on the Figure in dark).
Strains in the protrusion area reach values exceeding 2 thus allowing to suppose an essential cold work hardening in this zone. For example, Figure 5 shows the distribution of equivalent strains from 0.025 to 0.18 when stresses considerably grow in plasticity zone (from 100 to $240 \mathrm{MPa}$ ) or in other words, when a noticeable hardening takes place.



Figure 5. Distribution of equivalent strains corresponding to a significant hardening of the material.

The projecting upwards rectangular parallelogram is pressed to the level of the upper surface while this level itself rises by $0.0022 \mathrm{~m}$ due to redistribution of material to the area not affected by punch pressure. The finite element mesh refinement leads to a noticeable correction of results relating to equivalent plastic strain and punch displacement.

After complete flattening of the protrusion the second body turned to the same form as the first body, but of increased height taking into account the volume of the flattened protrusion. Geometrically, based on the ratio of the areas of the top of the protrusion and the plane on which the protrusion is located, the volume of protrusion material is sufficient to increase the height of the body by $3.6 \mathrm{~mm}$. In calculations the displacement of the upper plane after deformation of the first body was $1.52 \mathrm{~mm}$, that is, assuming equal displacement of the parts identical for two bodies, the increase in height of the second body after deformation as compared with the first one has to be not $3.6 \mathrm{~mm}$, but $3.6-1.52=2.08 \mathrm{~mm}$. This very value was received in numerical solution for the second body $10-7.78=2.22 \mathrm{~mm}$ with the accuracy of $7 \%$ since the initial height of the protrusion was $10 \mathrm{~mm}$, and the displacement of the deforming plane to complete crushing of the protrusion made $7.78 \mathrm{~mm}$ (see Table 1). Thus the received values of displacements for the punch confirm that the plastic deformation was calculated taking into account volumetric invariance, and compression of the first body by $1.52 \mathrm{~mm}$ is made within elasticity.

So it can be seen that complete flattening is possible at condition of uneven tri-axial compression provided that the material does not have an opportunity to widen in any direction. The asperities usually differ in size and the state mentioned above can take place only with such pressure with which asperities of all sizes in the considered area are flattened simultaneously. It occurs possibly at the close contact approaching to the perfect 
one when the real contact area comes to, say, $80-90 \%$ of the nominal one. Can asperities on asperities be completely flattened? No, as at flattening of a small asperity the material of underlying big asperity always has space where to move and the small asperity will preserve to some extent. To strengthen the above let us consider the following example.

\section{Deformation of truncated pyramids}

The result obtained in the previous section testifies to impossibility of full flattening of asperities. At the same time, it is not clear whether asperities of higher level in multilevel or fractal models of roughness are completely flattened. For this purpose, let us consider problems of deformation of two truncated pyramids - regular square and doubled. The regular square truncated pyramid represents an asperity model, and doubled one - model of asperity containing asperities of higher level.

\subsection{Regular pyramid model}

The truncated regular right square pyramid made of copper UNS C12500 with the base size $1 \mathrm{~m} \times 1 \mathrm{~m}$ and height of $1 \mathrm{~m}$ is deformed. The upper surface of the pyramid has the size of $0.1 \mathrm{~m} \times 0.1 \mathrm{~m}$. It is subjected to pressure up to $100 \mathrm{MPa}$ by the punch in the form of a rectangular parallelepiped with the size $1.4 \mathrm{~m} \times 1.4 \mathrm{~m} \times$ $0.4 \mathrm{~m}$. Punch material is hypothetical, elastic, with the modulus of elasticity of $2 \cdot 10^{18} \mathrm{~Pa}$ and Poisson's ratio of 0.3 , that is the deformation of the punch is considered insignificant. Lateral surfaces of the punch are fixed against displacements in $x_{1}$ and $x_{2}$ directions. Friction is neglected.

Let us consider 3D mathematical model of the contact of the rectangular parallelepiped and truncated pyramid with volumes $V_{1}$ and $V_{2}$, bounded by surfaces $S_{1}$ and $S_{2}$, respectively. We designate for the parallelepiped an upper flat surface $S_{1}^{1}$ and the rest of the surface $S_{1}^{2}$, so that $S_{1}=S_{1}^{1} \cup S_{1}^{2}$. For the pyramid we allocate in the surface $S_{2}$ the contacting surface $S_{2}^{1}$, the surface of the bottom base $S_{2}^{3}$, and the rest of the surface $S_{2}^{2}$, so that $S_{2}=S_{2}^{1} \cup$ $S_{2}^{2} \cup S_{2}^{3}$. The bottom base of the pyramid $S_{2}^{3}$ is fixed in all axes. The surface $S_{1}^{1}$ is affected by external pressure $P$.

The mathematical model of this problem comprises the equations (1)-(6) with the following boundary conditions

$$
\begin{gathered}
\sigma_{33}=P, x_{3} \in S_{1}^{1}, \\
u_{1}\left(x_{2}\right)=0, u_{2}\left(x_{1}\right)=0, x_{1}, x_{2} \in S_{1}^{2}, \\
u_{i}\left(x_{i}\right)=0, x_{i} \in S_{2}^{3} .
\end{gathered}
$$

ANSYS contact elements of CONTA174 type cover surfaces $S_{2}^{1}$ and $S_{2}^{2}$, and elements of TARGE170 type cover the bottom surface of the punch. The pyramid is meshed with hexahedral finite elements SOLID186 of uniform height (see Figure 6(a)).

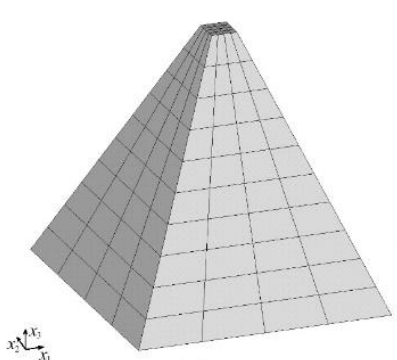

(a)

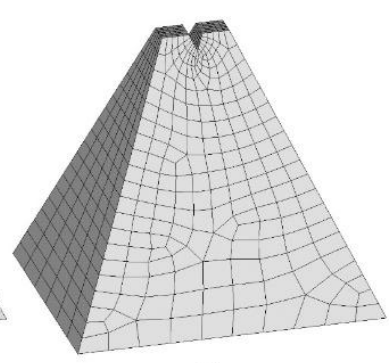

(b)
Figure 6. Finite element mesh of models: (a) regular square pyramid, (b) doubled pyramid.

The pressure is applied evenly in 400 substeps. Together with the meshing uniform by height it enables tracing, based on the graphs inclination, the parameters change rate for each row of finite elements. Rows are numbered starting from the top of the pyramid.

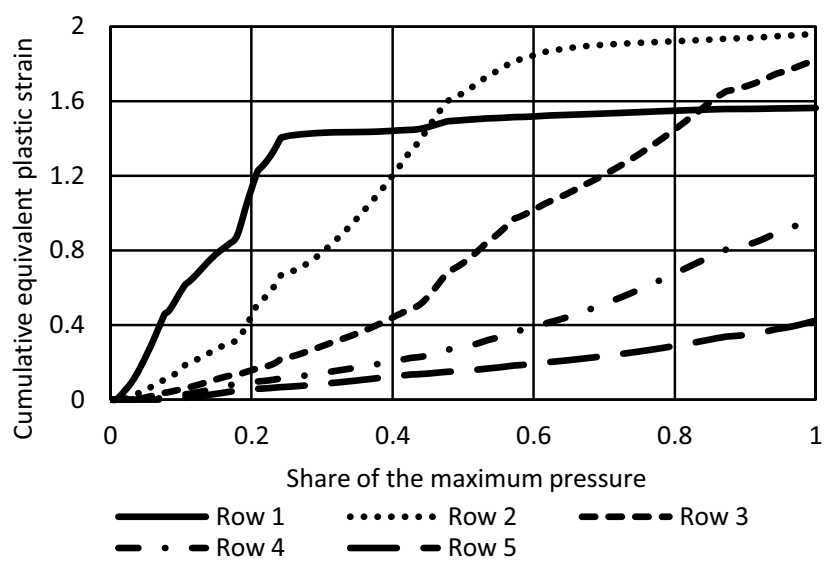

Figure 7. Cumulative equivalent plastic strain in the lower node of the finite element from the corresponding row of elements.

By results of the calculation (see Figure 7), it can be seen that of all elements of the pyramid the equivalent plastic strain growth rate is maximal for the first row elements. However, the strain growth with the maximal rate for the first row elements sharply stops when about $25 \%$ of the load is applied. The further strain growth is hardly noticeable. It is due to the upper row of elements is completely pressed into the underlying layer of elements at that moment (see Figure 8). Slowing the rate of strain growth can be explained with the fact that the first row of elements obtained higher hardening than the elements of the underlying second row and therefore stresses arising at this stage cause primarily strains of the less strengthened second row. For the first row in addition to weak deformation there is also a movement of elements downwards.

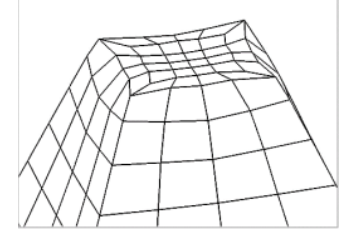

Figure 8. The first row of elements is pressed into elements of the second row at $25.4 \%$ of the maximum load.

When $57 \%$ of the maximum load is achieved, the intensive strain growth of the second row elements again 
stops because the first and second rows elements are fully pressed into the position of the third row elements.

Figure 9 shows the change in the maximal increment of equivalent plastic strains in the deforming body for each substep. In general, as it is possible to assume from the pyramid form, the decrease trend in strain increment values can be seen. Irregularity can be explained with features of the numerical implementation, such as discrete contact and a variety of the form of deformed finite elements.

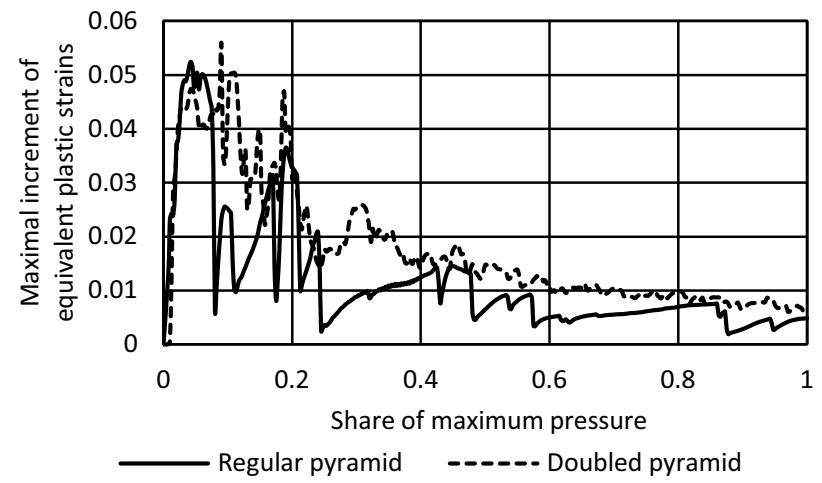

Figure 9. Maximal increment of equivalent plastic strains in the deforming body.

As it can be seen from Figure 10, the nodes of the top base move downwards within each row of elements with a constant rate. This rate decreases at indentation of one row into another row.

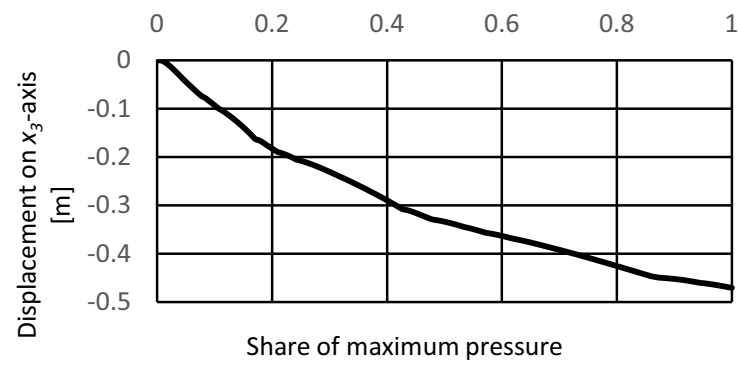

Figure 10. Displacement on $x_{3}$-axis of one of the top base nodes.

The increased hardening of the material in a row closer to the top of the pyramid in relation to the material of underlying rows showed a potential possibility of preserving the rough profile of overlying rows at further deformation of the pyramid. This rough profile may be initial or created at the contact deformation. On the other hand, the rough profile of an asperity, which is a roughness of the second level, is similar to a pyramid with an uneven surface. Preservation of such rough profile at deformation leads in turn to decrease in the real contact area. In the following problem such behavior is shown for a valley at the top of the doubled pyramid.

\subsection{Deformation of the doubled pyramid}

The model represents the figure consisting of two truncated square pyramids, same as the pyramid in the above problem, joined in one body. The one of corners of the first pyramid base forms the point of reference. The second pyramid is shifted along $x_{1}$-axis by $0.15 \mathrm{~m}$. Thus a valley is created between top surfaces of the pyramids $0.05 \mathrm{~m}$ wide and $0.0 \overline{5} \mathrm{~m}$ deep.

Here the figure of the model in a whole, that is the doubled pyramid, may be seen as an approximation model of asperity of the first level, and the valley between tops as a model of asperities of the second level actually existing on the first level asperity.

Mathematical model, material properties, the load, and solution options are the same as in the previous problem. The model is also meshed with hexahedral finite elements allowing to decrease numerical error. At the same time, the mesh is somewhat denser (see Figure 6(b)).

The numerical results are shown in Figure 9, 11-13. Due to the three-dimensional nature of the problem, the valley is deformed with a tendency to decrease its depth and to bring the opposing walls closer. When the top nodes were displaced $0.098 \mathrm{~m}$ downward, the opposite walls were closed at the ends of the valley.

After the full load applied and a considerable crushing occurred, it can be seen that irrespective of the pressure, the valley remains and creates a steady gap between the punch and the surface being deformed (Figure 11). The maximal depth of the valley is $0.0186 \mathrm{~m}$, or more than $30 \%$ of the initial. Growth rate of the valley depth sharply decreases after flattening the tops of the pyramid (at about $20 \%$ of the load) (Figure 12). As the valley tends to close, the presence of friction would apparently increase the gap.



(a)

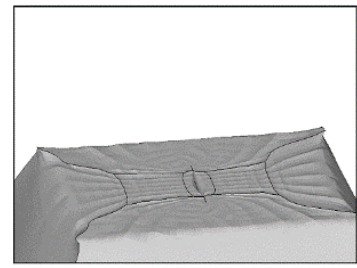

(b)

Figure 11. Valley of the double pyramid: before (a) and after (b) deformation.



Figure 12. Change in the valley depth.

Figure 13 shows that the cumulative equivalent plastic strains and corresponding hardening of material in the valley area are not large. The layer of strengthened 
material and the maximal stresses are distributed as a belt at some distance under the valley. It happens because of both the form of the model being deformed and elasticplastic behavior of the material that allows hardening.

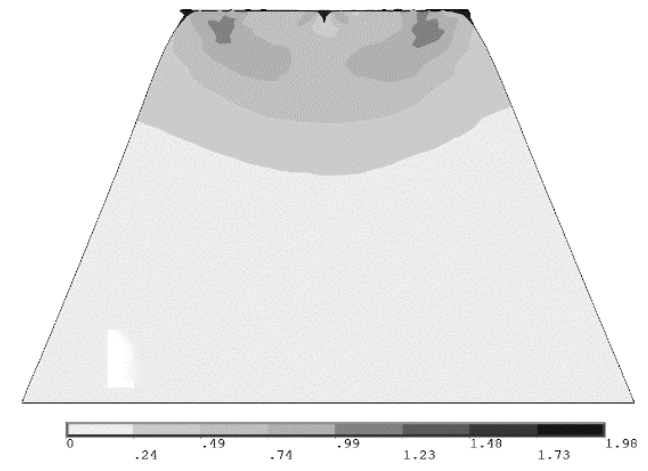

Figure 13. The cumulative equivalent plastic strains at the half of the maximum load. A section of the pyramid in half in $x_{1} x_{3}$ plane is shown.

Change in the maximal increment of the equivalent plastic strains (see Figure 9) is similar to that in the regular pyramid problem. The curve is less rugged because of the refined mesh.

\section{Discussing the results}

Solution of the deformation problem for the model with the protrusion made of isotropic hardening material has shown that on condition of uneven tri-axial compression when there is no free space for the material to move in the lateral directions the protrusions on surface are completely flattened at certain pressure. In this case the surface is considerably strengthened although at some depth a layer is formed, even less hardened compared to the model without the protrusion.

But asperities material on a real surface, when the contact is not close to perfect, have a space where to move in the lateral directions, as the contact of the asperities generally happens serially, not concurrently. Then the asperities are not under condition of uneven triaxial compression. Therefore, complete flattening of asperities of the rough surface will not occur even if we assume the absence of such preventing from flattening factors as indentation size effect, initial cold work hardening and friction. It again emphasizes the role of asperities form in modeling the contact of rough bodies.

While deforming with a plane punch all domains protruding above the nominal level of rough surface in which hardening exceeds the hardening of material at the nominal level, will be further deformed slower, than the material at the nominal level, and can create a rough profile. Perfectly plastic material would apparently behave in another way, that is would be completely deformed adapting to the punch. In case of elastic-plastic material behavior, the forming of rough profile happens because of inhomogeneous spatial distribution of stresses and is possible only in combination with a "favorable" form of the objects being deformed. It seems that the models of asperities (for example, set of several pyramids) possess such a form, especially in case of inclusion asperities of the second level in them.
At the beginning when the top of the asperity is touched the maximal stresses is in the top area. In the course of deformation there comes a moment when stresses in top decrease to the level below the actual yield stress and the plastic deformation continues in deeper areas. So the strengthened top begins to hold its profile. Thus, the rough surface profile can be formed and supported at severe deformation in the near-surface layer of the body. At sufficient hardening of deeper layers and at the appropriate current surface profile, which leads to the necessary redistribution of pressure, the plastic deformation of the top can resume.

So, the assumption of perfect contact in the area of direct contact of asperities is mistaken. The asperities are not flattened completely because of the roughness of the second and higher levels, and also because of the hardening.

Complete flattening of asperities might perhaps occur only at a very high pressure when the displacements exceed several times the size of asperities being smoothed. It seems that such pressure cause plastic deformations noticeable with the naked eye and is not often met in assemblies.

Let us note that we did not consider the compliance of the bottom surface of the pyramids taking place for a real near-surface layer of roughness. This assumption could somewhat promote the smoothing of the rough profile. The indentation size effect and friction at their inclusion in solution could have the opposite effect. However, the above factors do not result in a qualitative change.

\section{Conclusion}

The results showed that, in the case of elastic-plastic behavior of material, even under considerable pressure and strains, complete flattening of asperities will not occur in the general case, because there will be no confined compression. The relief will contain asperities of different scale. The asperities of higher level will change the real contact area. The calculations carried out in the first approximation show the tendency for it to decrease $[7,16]$. The quantitation of influence of higher level asperities at elastic-plastic material behavior is a key issue for future research. Perhaps for some cases, higher level asperities can even be neglected. Nevertheless, multilevel or fractal descriptions of roughness, apparently, can be considered more truly characterizing the processes in contact.

One of the most important in determining the degree of influence of higher level asperities will be a combination of geometric shape of rough surface with the elastic-plastic properties of the material, especially with its stress-strain curve. In view of the geometric complexity of contacting bodies and the nonlinear behavior of materials, the use of 3D-models and numerical methods, finite element method in particular, could be promising here.

Moreover, asperities of higher level can play a special role if we take into account the change of mechanical properties of near-surface material due to the indentation size effect. This will be explored as the future 
development of the finite-element rough contact micromodel of the author, described in [7].

\section{References}

1. A.L. Wang, J.F. Zhao., Sci China Technol Sci 53(7), 1798-1808 (2010)

2. M.M. Yovanovich, IEEE Trans Compon Packaging Technol 28(2), 182-206 (2005)

3. Y. Chen, X. Huang, R. Kang, Chin J Aeronaut 25(4), 584-592 (2012)

4. J.F. Archard, Proc R Soc Lond A Math Phys Sci 243(1233), 190-205 (1957)

5. G. Zavarise, M. Borri-Brunetto, M. Paggi, Wear 257(3-4), 229-245 (2004)

6. M.V. Murashov, S.D. Panin, Proceedings of the International heat transfer conference IHTC14, 6, 387-392 (2010)

7. M.V. Murashov, S.D. Panin, Int J Heat Mass Transf 90, 72-80 (2015)

8. W. Yan, K. Komvopoulos, J Appl Phys 84, $3617-$ 3624 (1998)

9. M.S. Bobji, S.K. Biswas, J.B. Pethica, Appl Phys Lett 71, 1059-1061 (1997)

10. C. Ji, H. Zhu, W. Jiang, Chin J Mech Eng 26(1), 128136 (2013)

11. C.K. Bora, E.E. Flater, M.D. Street, et al, Tribol Lett 19(1), 37-48 (2005)

12. W. Pan, X. Li, N. Guo, et al, Adv Mech Eng 9(3), 111 (2017)

13. M. Ciavarella, V. Delfine, G.Demelio, J Mech Phys Solids 54, 2569-2591 (2006)

14. M. Bahrami, M.M. Yovanovich, J.R. Culham, Int J Heat Mass Transf 48(16), 3284-3293 (2005)

15. M.K. Thompson, $\mathrm{PhD}$ thesis. Cambridge, MA, USA: Massachusetts Institute of Technology(2007)

16. S. Lee, Y.H. Jang, W. Kim, J Appl Phys 103, 074308 (2008)

17. M.H. Müser, W.B. Dapp, R. Bugnicourt, et al, Tribol Lett 65, 118 (2017)

18. G. Carbone, F. Bottiglione, J Mech Phys Solids 56, 2555-2572 (2008)

19. C. Yang, B. Persson, J PhysCondens Matter 20, 215214 (2008)

20. Y. Waddad, V. Magnier, Ph. Dufrénoy, et al, Tribol Lett 65, 155 (2017)

21. M. Liu, H. Proudhon, Mech Mater 103, 78-86 (2016)

22. J.A. Greenwood, Br J Appl Phys 17(12), 1621-1632 (1966)

23. A.J.W. Moore, Proc R Soc Lond A Math Phys Eng Sci 195, 231-244 (1948)
24. K.A. Kochergin, Contact Welding (Machine building Publ., Leningrad, Russia, 1987)

25. S. Cai, PhD thesis. Columbus, Ohio, USA: The Ohio State University (2008)

26. H. Ghaednia, X. Wang, S. Saha, et al, Appl Mech Rev 69(6), 060804 (2017)

27. H. Hertz, J Reine Angew Math 92(1), 156-171 (1882)

28. H. Songa, A.I. Vakisb, X. Liuc, et al, J Mech Phys Solids 106, 1-14 (2017)

29. F. Sun, E. Van der Giessen, L. Nicola, Wear 296(12), 672-680 (2012)

30. L. Kogut, I. Etsion, J Appl Mech 69(5), 657-662 (2002)

31. R.L. Jackson, I. Green, J Tribol 127(2), 343-354 (2005)

32. P. Sahoo, B. Chatterjee, Eng 2, 205-211 (2010)

33. H. Ghaednia, A. Rostami R.L. Jackson, IEEE 58th Holm Conference on Electrical Contacts, Protland, OR, USA, 1-7 (2012)

34. S.S. Wadwalkar, R.L. Jackson, L. Kogut, Proc Inst Mech Eng J 224, 1091-1102 (2010)

35. A. Goedecke, R.L. Jackson, R. Mock, Wear 268, 1285-1294 (2010)

36. J.A. Greenwood, J.B.R. Williamson. Proc R Soc Lond A Math Phys Sci 295, 300-319 (1966)

37. X. Zhang, P. Cong, S. Fujiwara, et al, Int J Heat Mass Transf 47(5), 1091-1098 (2004)

38. Y. Zhao, L. Chang, J Tribol 123, 857-864 (2001)

39. P. Sahoo, A. Banerjee, J Phys D Appl Phys 38, 40964103 (2005)

40. P. Sahoo, J Phys D Appl Phys 39, 2809-2818 (2006)

41. M. Ciavarella, J.A. Greenwood, M. Paggi, Wear 265, 729-734 (2008)

42. M. Paggi, M. Ciavarella, Wear 268(7), 1020-1029 (2010)

43. I. Manika, J. Maniks, Acta Mater 54(8), 2049-2056 (2006)

44. M.V. Murashov, Y.V. Kornev, Tech Phys 84(3), 75$81(2014)$

45. Y.G. Kalpin, V.I. Perfilov, P.A. Petrov, et al, Resistance to deformation and plasticity of metals treated by pressure (Machine building Publ., Moscow, Russia, 2011)

46. L. Wang, H. Liu, J. Zhang, et al. Precis Eng 37(4), 817-824 (2013)

47. G.E. Mase. Schaum's outline of theory and problems of continuum mechanics. (New York: McGraw-Hill, 1970)

48. T.J.R. Hughes. In: Nemat-Nasser S, Asaro RJ, Hegemier GA (eds) Mechanics of elastic and inelastic solids 6, 30-57 (Dordrecht, Springer, 1984) 